\title{
Mathematical modelling of phenotypic selection within solid tumours
}

\author{
Mark A.J. Chaplain ${ }^{1}$, Tommaso Lorenzi ${ }^{2}$, Alexander Lorz ${ }^{3}$, and \\ Chandrasekhar Venkataraman ${ }^{4}$ \\ 1 School of Mathematics and Statistics, University of St Andrews, St Andrews \\ KY16 9SS, United Kingdom, majc@st-andrews.ac.uk \\ 2 School of Mathematics and Statistics, University of St Andrews, St Andrews \\ KY16 9SS, United Kingdom, t147@st-andrews.ac.uk \\ 3 CEMSE Division, King Abdullah University of Science and Technology \\ (KAUST), Thuwal, Saudi Arabia \& Sorbonne Universités, UPMC Univ Paris \\ 06, UMR 7598, Laboratoire Jacques-Louis Lions, Paris, France, \\ Alexander.Lorz@kaust. edu.sa \\ 4 School of Mathematics and Statistics, University of St Andrews, St Andrews \\ KY16 9SS, United Kingdom, cv28@st-andrews.ac.uk
}

\begin{abstract}
We present a space- and phenotype-structured model of selection dynamics between cancer cells within a solid tumour. In the framework of this model, we combine formal analyses with numerical simulations to investigate in silico the role played by the spatial distribution of oxygen and therapeutic agents in mediating phenotypic selection of cancer cells. Numerical simulations are performed on the 3D geometry of an in vivo human hepatic tumour, which was imaged using computerised tomography. Our modelling extends our previous work in the area through the inclusion of multiple therapeutic agents, one that is cytostatic, whilst the other is cytotoxic. In agreement with our previous work, the results show that spatial inhomogeneities in oxygen and therapeutic agent concentrations, which emerge spontaneously in solid tumours, can promote the creation of distinct local niches and lead to the selection of different phenotypic variants within the same tumour. A novel conclusion we infer from the simulations and analysis is that, for the same total dose, therapeutic protocols based on a combination of cytotoxic and cytostatic agents can be more effective than therapeutic protocols relying solely on cytotoxic agents in reducing the number of viable cancer cells.
\end{abstract}

\section{Introduction}

In this work, we extend a space- and phenotype-structured model of selection dynamics in a solid tumour that we proposed in [4,5]. Our model consists of a nonlinear integro-differential equation (IDE) for the spatiotemporal evolution of the phenotypic distribution of cancer cells coupled to a system of nonlinear partial differential equations (PDEs) for the dynamics of abiotic factors. A novel feature of this work, over [4], is that we consider multiple spatially distributed therapeutic agents. Through the coupling of formal analyses and numerical simulations, we show that spatial variations in abiotic 
factors promote the formation of local niches and lead to the selection of different phenotypic variants, in agreement with the viewpoint of cancer as an eco-evolutionary process [6]. The modelling carried out in the present work allows us to consider optimisation of treatment protocols. In particular, our results suggest that, for the same total dose, therapeutic protocols based on the delivery of cytotoxic drugs in combination with cytostatic agents can be more effective than therapeutic protocols relying solely on cytotoxic drugs in reducing the number of viable cancer cells.

\section{Model description}

We identify the tumour geometry with a spatial domain $\Omega \subset \mathbb{R}^{3}$. At any time instant $t \geq 0$, we characterise the state of each cancer cell in the tumour by means of a pair $(\mathbf{x}, y) \in \Omega \times[0,1]$. The vector $\mathbf{x} \in \Omega$ identifies the spatial position of the cell and the scalar variable $y \in[0,1] \subset \mathbb{R}$ stands for the normalised expression level of a hypoxia-responsive gene [8]. Cells within the tumour proliferate and die due to competition for limited space. Moreover, both a cytotoxic drug, which acts by increasing the cell death rate, and a cytostatic drug, which acts by reducing the cell proliferation rate, can be administered. We assume increasing values of the phenotypic state $y$ to be correlated with a progressive switch towards a hypoxic phenotype which, in turn, implies a progressive reduction in the proliferation rate [1]. Additionally, given that cytotoxic agents target mostly rapidly proliferating cells, we assume that higher values of the phenotypic state $y$ correspond with higher levels of resistance to the cytotoxic drug [3].

Given the local population density $n(t, \mathbf{x}, y)$, we compute the cell density and the mean cell phenotypic state at time $t$ and position $\mathbf{x}$ as follows

$$
\rho(t, \mathbf{x})=\int_{0}^{1} n(t, \mathbf{x}, y) \mathrm{d} y \quad \text { and } \quad \mu(t, \mathbf{x})=\frac{1}{\rho(t, \mathbf{x})} \int_{0}^{1} y n(t, \mathbf{x}, y) \mathrm{d} y .
$$

Finally, we introduce the functions $s(t, \mathbf{x}) \geq 0, c_{1}(t, \mathbf{x}) \geq 0$ and $c_{2}(t, \mathbf{x}) \geq 0$ to model the local concentration of oxygen, cytotoxic drug and cytostatic drug at position $\mathbf{x}$ and time $t$, respectively.

\subsection{Dynamics of cancer cells}

The dynamics of the local population density $n(t, \mathbf{x}, y)$ is governed by the following nonlinear IDE

$$
\frac{\partial n}{\partial t}(t, \mathbf{x}, y)=R\left(y, \rho(t, \mathbf{x}), s(t, \mathbf{x}), c_{1}(t, \mathbf{x}), c_{2}(t, \mathbf{x})\right) n(t, \mathbf{x}, y) .
$$

In Eq. (2), the functional $R\left(y, \rho, s, c_{1}, c_{2}\right)$ represents the fitness of cells in phenotypic state $y$ at position $\mathbf{x}$ and time $t$ (i.e. the fitness landscape of 
the tumour), given the local environmental conditions determined by the cell density $\rho(t, \mathbf{x})$ and the concentrations of abiotic factors $s(t, \mathbf{x}), c_{1}(t, \mathbf{x})$ and $c_{2}(t, \mathbf{x})$. We define the fitness landscape of the tumour as

$$
\begin{aligned}
R\left(y, \rho, s, c_{1}, c_{2}\right)= & \underbrace{f(y)}_{\begin{array}{c}
\text { proliferation } \\
\text { in hypoxic conditions }
\end{array}}+\underbrace{r(y, s)\left(1-k_{2}\left(c_{2}\right)\right)}_{\begin{array}{c}
\text { proliferation } \\
\text { in oxygenated environments }
\end{array}} \\
& -\underbrace{k_{1}\left(y, c_{1}\right)}_{\begin{array}{c}
\text { death due to } \\
\text { cytotoxic drug }
\end{array}}-\underbrace{d \rho(t, \mathbf{x})}_{\begin{array}{c}
\text { death due to } \\
\text { competition for space }
\end{array}} .
\end{aligned}
$$

Building upon the considerations and the modelling strategies presented in [4], we introduce the following definitions:

$$
\begin{gathered}
f(y)=\zeta\left[1-(1-y)^{2}\right], \quad r(y, s)=\gamma_{s} \frac{s(t, \mathbf{x})}{\alpha_{s}+s(t, \mathbf{x})}\left(1-y^{2}\right), \\
k_{1}\left(y, c_{1}\right)=\gamma_{c_{1}} \frac{c_{1}(t, \mathbf{x})}{\alpha_{c_{1}}+c_{1}(t, \mathbf{x})}(1-y)^{2}, \quad k_{2}\left(c_{2}\right)=\gamma_{c_{2}} \frac{c_{2}(t, \mathbf{x})}{\alpha_{c_{2}}+c_{2}(t, \mathbf{x})} .
\end{gathered}
$$

The biological meaning of the different parameters are summarised in Table 1. The function $0 \leq k_{2}\left(c_{2}\right) \leq 1$ measures the percentage reduction in the proliferation rate of cancer cells caused by the cytostatic drug. Since the efficacy of many cytostatic drugs is directly linked with adequate oxygen tension [2], we make the prima facie assumption that the cytostatic drug reduces the proliferation rate in oxygenated environments, whereas it does not affect the proliferation rate under hypoxic conditions. For this reason, the term $f(y)$ is not multiplied by the factor $\left(1-k_{2}\left(c_{2}\right)\right)$. Moreover, we assume the function $k_{2}$ to be increasing in the drug dose $c_{2}$. A detailed discussion of the biological assumptions that underlie the definitions of the other functions can be found in [4].

\subsection{Dynamics of abiotic factors}

The abiotic factors diffuse in space, decay over time and are consumed by cells. We note that the dynamics of abiotic factors is faster than cellular proliferation and death [9]. From a mathematical viewpoint, this means that we can assume oxygen and the drugs to be in quasi-stationary equilibrium. In this setting, the dynamics of the functions $s(t, \mathbf{x}), c_{1}(t, \mathbf{x})$ and $c_{2}(t, \mathbf{x})$ are described by the following elliptic PDEs which are coupled to the IDE (2)

$$
\begin{gathered}
\beta_{s} \Delta s(t, \mathbf{x})=\eta_{s} \int_{0}^{1} r(y, s) n(t, \mathbf{x}, y) \mathrm{d} y+\lambda_{s} s(t, \mathbf{x}), \\
\beta_{c_{1}} \Delta c_{1}(t, \mathbf{x})=\eta_{c_{1}} \int_{0}^{1} k_{1}\left(y, c_{1}\right) n(t, \mathbf{x}, y) \mathrm{d} y+\lambda_{c_{1}} c_{1}(t, \mathbf{x}),
\end{gathered}
$$


and

$$
\beta_{c_{2}} \Delta c_{2}(t, \mathbf{x})=\eta_{c_{2}} \int_{0}^{1} k_{2}\left(c_{2}\right) n(t, \mathbf{x}, y) \mathrm{d} y+\lambda_{c_{2}} c_{2}(t, \mathbf{x}) .
$$

The biological meanings of the different parameters are summarised in Table 1 and further details can be found in [4]. Concentrating on the biological scenario whereby the tumour is avascular and the concentrations of abiotic factors in the medium surrounding the tumour are constant in time, we choose the following boundary conditions for equations (6)-(8)

$$
s(\cdot, \mathbf{x})=S(\mathbf{x}), \quad c_{1}(\cdot, \mathbf{x})=C_{1}(\mathbf{x}) \quad \text { and } \quad c_{2}(\cdot, \mathbf{x})=C_{2}(\mathbf{x}) \quad \forall \mathbf{x} \in \partial \Omega .
$$

\section{Formal analysis of phenotypic selection}

We denote the local cell density and the dominant phenotypic state (i.e. the phenotypic state $y$ that maximises the cellular fitness at position $\mathbf{x}$ ) at equilibrium as $\bar{\rho}(\mathbf{x})$ and $\bar{y}(\mathbf{x})$, respectively. Moreover, we denote the steadystate distributions of abiotic factors as $\bar{s}(\mathbf{x}), \bar{c}_{1}(\mathbf{x})$ and $\bar{c}_{2}(\mathbf{x})$. By means of the formal arguments used in [4], one finds

$$
\bar{\rho}(\mathbf{x})=\frac{1}{d}\left[A_{\bar{s}, \bar{c}_{2}}(\mathbf{x})-A_{\bar{c}_{1}}(\mathbf{x})+\frac{\left(\zeta+A_{\bar{c}_{1}}(\mathbf{x})\right)^{2}}{\zeta+A_{\bar{s}, \bar{c}_{2}}(\mathbf{x})+A_{\bar{c}_{1}}(\mathbf{x})}\right]
$$

and

$$
\bar{y}(\mathbf{x})=\frac{\zeta+A_{\bar{c}_{1}}(\mathbf{x})}{\zeta+A_{\bar{s}, \bar{c}_{2}}(\mathbf{x})+A_{\bar{c}_{1}}(\mathbf{x})},
$$

where

$$
A_{\bar{s}, \bar{c}_{2}}(\mathbf{x})=\gamma_{s}\left(1-k_{2}\left(\bar{c}_{2}\right)\right) \frac{\bar{s}(\mathbf{x})}{\alpha_{s}+\bar{s}(\mathbf{x})} \text { and } A_{\bar{c}_{1}}(\mathbf{x})=\gamma_{c_{1}} \frac{\bar{c}_{1}(\mathbf{x})}{\alpha_{c_{1}}+\bar{c}_{1}(\mathbf{x})} .
$$

Such formal results are consistent with the asymptotic results presented in [7].

The expressions given by equations (10) and (11) demonstrate that the local cell density $\bar{\rho}$ and the dominant phenotypic state $\bar{y}$ at a certain position are determined by the concentrations of oxygen $\bar{s}$, cytotoxic drug $\bar{c}_{1}$ and cytostatic agent $\bar{c}_{2}$ at the same position. This is illustrated by the heat-maps in Figure 1, which relate to the parameter values given in Table 1 and

$$
\bar{c}_{1}=K C_{t o t}, \quad \bar{c}_{2}=(1-K) C_{t o t}, \quad K \in[0,1] .
$$

The heat-maps display the values of $\bar{\rho}$ and $\bar{y}$ as functions of the parameter $K$ in Eq.(12) and the oxygen concentration $\bar{s}$. We observe that, for sufficiently high oxygen levels, the local cell density is minimised for values of $K$ between 0.4 and 0.5 , which corresponds to the situation whereby the cytotoxic drug and the cytostatic agent are used in combination. 

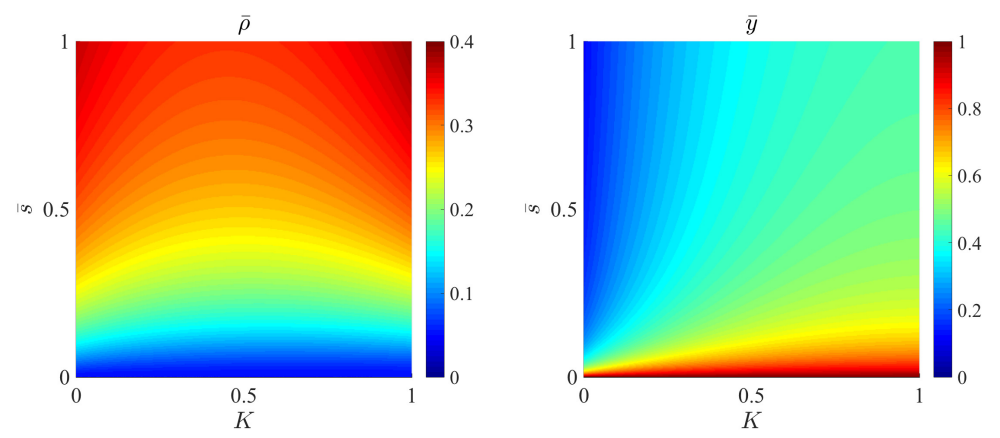

Figure1: Heat-maps showing the local cell density $\bar{\rho}$ and dominant phenotypic state $\bar{y}$ at equilibrium as functions of the parameter $K$ [cf. Eq.(12)] and the local concentration of oxygen $\bar{s}$. For sufficiently high oxygen levels, the local cell density is minimised for values of $K$ between 0.4 and 0.5 , which corresponds to the situation whereby the cytotoxic drug and the cytostatic agent are used in combination.

\section{Numerical solutions}

In this section we report on simulations of the model system (2), (6), (7) and (8). For the spatial domain $\Omega$ we consider a tetrahedral discretisation of the real geometry of a human hepatic tumour obtained from the 3D-IRCADb01 database (http://www.ircad.fr/). We make use of the following boundary conditions for $s, c_{1}$ and $c_{2}$ :

$$
S(\mathbf{x})=S_{0} \quad \forall \mathbf{x} \in \partial \Omega
$$

and

$$
C_{1}(\mathbf{x})=K C_{t o t}, \quad C_{2}(\mathbf{x})=(1-K) C_{t o t} \quad \forall \mathbf{x} \in \partial \Omega \quad \text { with } \quad K \in[0,1]
$$

The parameter $C_{t o t}$ measures the total delivered drug dose. To carry out numerical simulations, we tune the value of the parameter $K$ and we use the values given in Table 1 for the other parameters of the model. Further details on the tumour geometry and the model parametrisation can be found in [4].

For the elliptic PDEs, we use a $\mathbb{P}^{1}$ finite element method and treat the nonlinear terms explicitly using the values from the previous time-step. For the IDE, we use an IMEX Euler method in which the nonlinear terms are treated explicitly. The mesh that we employ has 9932 DOFs and the timestep we use correspond to $1 \times 10^{3}$ with a final time of $5 \times 10^{6}$, by which 
Table1: Nondimensionalised parameter values

$\begin{array}{cll}\text { Parameter } & \text { Biological meaning } & \text { Value } \\ \alpha_{c_{1}} & \text { Michaelis-Menten constant of cytotoxic drug } & 0.2 \\ \alpha_{c_{2}} & \text { Michaelis-Menten constant of cytostatic drug } & 10^{-2} \\ \alpha_{s} & \text { Michaelis-Menten constant of oxygen } & 0.2344 \\ \beta_{c_{1}}, \beta_{c_{2}} & \text { Diffusion coefficients of cytotoxic and cytostatic drug } & 5 \\ \beta_{s} & \text { Diffusion coefficient of oxygen } & 20 \\ \gamma_{c_{1}} & \text { Maximum cell death rate due to cytotoxic drug } & 1.8 \times 10^{-4} \\ \gamma_{c_{2}} & \text { Maximum percentage reduction in cell proliferation rate due to cytostatic drug } 0.8 \\ \gamma_{s} & \text { Maximum cell proliferation rate in oxygenated environments } & 1.5 \times 10^{-5} \\ \zeta & \text { Maximum cell proliferation rate under hypoxic conditions } & 1 \times 10^{-6} \\ d & \text { Rate of cell death due to competition for space } & 2 \times 10^{-5} \\ \eta_{c_{1}}, \eta_{c_{2}} & \text { Scaling factors for cell consumption of the drugs } & 400 \\ \eta_{s} & \text { Scaling factor for cell consumption of oxygen } & 3125.2 \\ \lambda_{c} & \text { Decay rate of cytotoxic drug } & 0.1 \\ \lambda_{s} & \text { Decay rate of oxygen } & 0.3 \\ C_{t o t} & \text { Total drug dose delivered } & 0.01 \\ S_{0} & \text { Oxygen concentration in the surrounding environment } & 1\end{array}$

time the numerical solutions are at steady state values in all the cases under consideration. The numerical method employed is detailed in [4].

Figure 2 summarises the numerical solutions at equilibrium for $K=0.6$ [cf. Eq.(13)]. We show a slice through the tumour in order to visualise the interior. We also post-process the computed values of $S_{h}, C_{1, h}$ and $C_{2, h}$ to evaluate the corresponding dominant phenotypic state $\bar{y}$ and the local cell density $\bar{\rho}$ according to Eqs.(10)-(11), which we compare with the computed mean phenotypic state $\mu_{h}$ and local cell density $\rho_{h}$. There is an excellent agreement between the numerical solutions at equilibrium and the predictions of our formal analyses. As the qualitative features of the numerical solutions for the other values of $K$ are similar to those of the numerical solutions obtained for $K=0.6$, we do not display the equilibrium spatial distributions for other values of $K$. However, in order to illustrate the variation in cell density and phenotypic state at equilibrium as we change $K$, in Figure 3 we report on the computed total cell number and mean phenotypic state at equilibrium in the tumour and on the tumour surface. The total number of cells and the surface cell number at equilibrium are minimised for $K \approx 0.8$ and $K \approx 0.4$, respectively. We note that this agrees with the results of the formal analysis illustrated by the heat-map in the left panel of Figure 1 as the concentrations of therapeutic factors and oxygen correspond to those used to make the plots of Figure 1 only at the boundary of the tumour.

Our results suggest that therapeutic protocols based on the delivery of lower doses of cytotoxic drugs in combination with cytostatic agents may be more effective than therapeutic protocols relying solely on higher doses of cytotoxic drugs in reducing the number of viable cancer cells. 


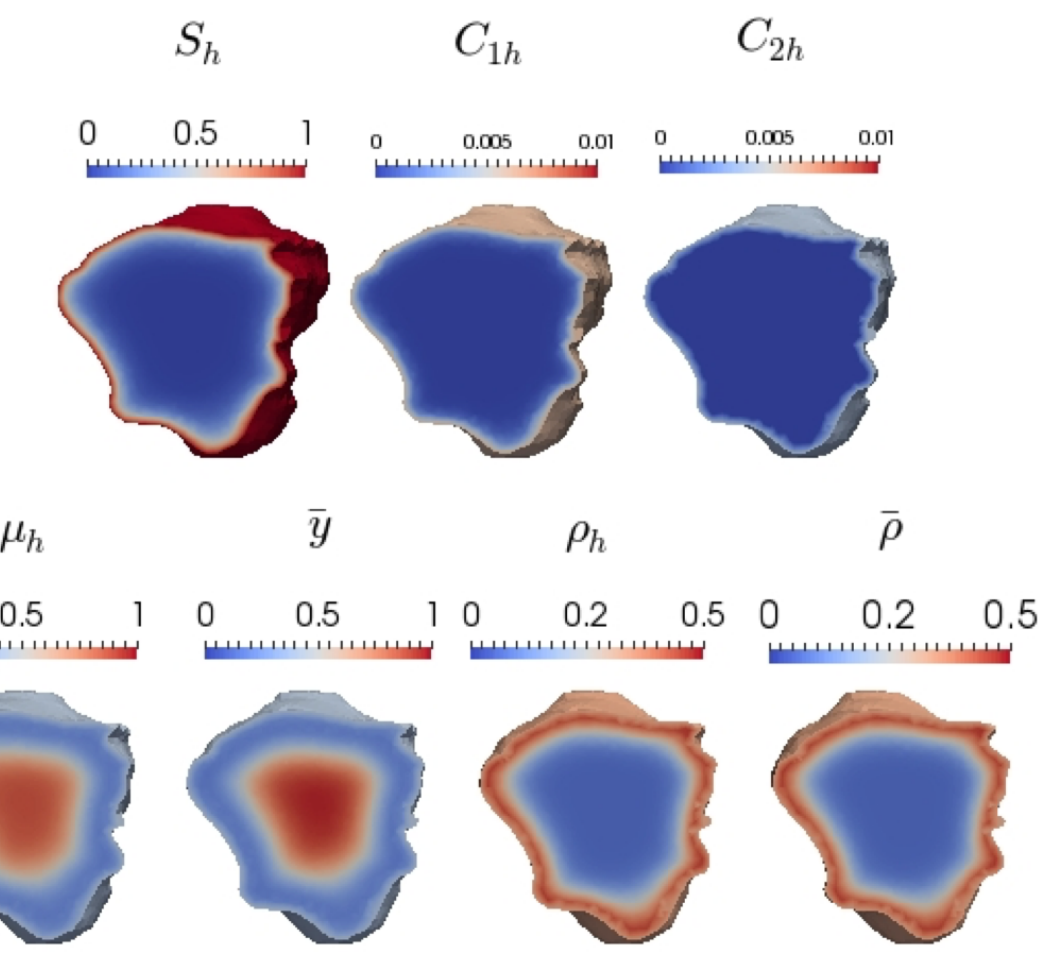

Figure2: Upper row: computed equilibrium distributions of oxygen $\left(S_{h}\right)$, cytotoxic drug $\left(C_{1 h}\right)$ and cytostatic drug $\left(C_{2 h}\right)$ for $K=0.6$. Lower row: computed mean phenotypic state $\left(\mu_{h}\right)$, predicted dominant trait $(\bar{y})$, computed $\left(\rho_{h}\right)$ and predicted $(\bar{\rho})$ local cell density at equilibrium for $K=0.6$. The results of the formal analysis agree with the results of numerical simulations.
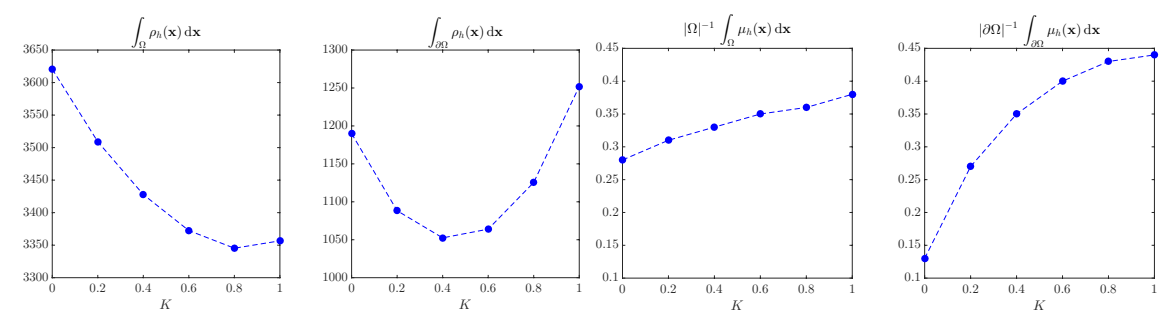

Figure3: Reading from left to right, computed total cell number, surface cell number, mean phenotypic state over the tumour and surface mean phenotypic state at equilibrium for different values of the parameter $K$. The total cell number over the tumour is minimised amongst the considered values by taking $K \approx 0.8$, whilst the surface cell number is minimised for $K \approx 0.4$. 


\section{References}

1. J. D. Gordan, J. A. Bertout, C.-J. Hu, J. A. Diehl, and M. C. Simon, Hif-2 $\alpha$ promotes hypoxic cell proliferation by enhancing c-myc transcriptional activity, Cancer Cell 11:4 (2007), 335-347.

2. C. Legendre, S. Avril, C. Guillet, and E. Garcion, Low oxygen tension reverses antineoplastic effect of iron chelator deferasirox in human glioblastoma cells, BMC cancer 16:1 (2016), 51.

3. M. C. Lloyd, J. J. Cunningham, M. M. Bui, R. J. Gillies, J. S. Brown, AND R. A. GATEnBy, Darwinian dynamics of intratumoral heterogeneity: not solely random mutations but also variable environmental selection forces, Cancer Research 76:11 (2016), 3136-3144.

4. T. Lorenzi, C. Venkataraman, A. Lorz, and M. A. Chaplain, The role of spatial variations of abiotic factors in mediating intratumour phenotypic heterogeneity, Submitted, Preprint available from http://hdl.handle.net/10023/10685 (2017).

5. A. Lorz, T. Lorenzi, J. Clairambault, A. Escargueil, And B. PerTHAME, Modeling the effects of space structure and combination therapies on phenotypic heterogeneity and drug resistance in solid tumors, Bulletin of Mathematical Biology 77:1 (2015), 1-22.

6. L. M. Merlo, J. W. Pepper, B. J. Reid, and C. C. Maley, Cancer as an evolutionary and ecological process, Nature Reviews Cancer 6:12 (2006), 924-935.

7. S. Mirrahimi And B. Perthame, Asymptotic analysis of a selection model with space, Journal de Mathématiques Pures et Appliquées 104:6 (2015), 11081118.

8. S. Strese, M. Fryknäs, R. Larsson, and J. Gullbo, Effects of hypoxia on human cancer cell line chemosensitivity, BMC Cancer 13:1 (2013), 1.

9. V. Walther, C. T. Hiley, D. Shibata, C. Swanton, P. E. Turner, and C. C. Maley, Can oncology recapitulate paleontology? Lessons from species extinctions, Nat Rev Clin Oncol 12:5 (2015), 273-285.

\section{Acknowledgments}

CV wishes to acknowledge partial support from the European Union's Horizon 2020 research and innovation programme under the Marie SklodowskaCurie grant agreement No 642866. AL was supported by King Abdullah University of Science and Technology (KAUST) baseline and start-up funds (BAS/1/1648-01-01 and BAS/1/1648-01-02). MAJC gratefully acknowledges support of EPSRC grant no. EP/N014642/1. 